\title{
QUEDARSE EN EL PUEBLO: SOLIDARIDADES FAMILIARES Y DESIGUALDADES DE GÉNERO en un ejido Cafetalero del Soconusco (Chiapas, México)
}

\section{Staying in the Village: Family Solidarities and Gender IneQualities in a Coffee Ejido of the Soconusco (Chiapas, Mexico)}

\author{
Alicia Rinaldy* \\ DOI: http://dx.doi.org/10.29043/liminar.v19i2.847
}

Resumen: En esta investigación se lleva a cabo una reflexión sobre el papel que juegan las solidaridades familiares y el sistema de género en los procesos de arraigo en los espacios rurales. En este artículo se dan a conocer dos casos etnográficos, dos trayectorias de vida, las de un hombre y de una mujer. Son productores de café al sur del Soconusco, en Chiapas, y pertenecen a una generación nacida en los años 1950 que vivió dos momentos históricos distintos: una primera socialización estructurada alrededor de la producción agrícola de la parcela y del ejido, y una nueva etapa a partir de los años 1990, cuando esta misma generación enfrentó procesos de desagrarización. El artículo revela el peso del género sobre las solidaridades familiares porque los hombres y las mujeres no heredan los mismos recursos ni ejercen las mismas obligaciones en las familias.

Palabras clave: comunidades rurales, ejidos, familia, desigualdades de género.

Abstract: We discuss in this paper the role played by family solidarities and the gender system in the processes involved in putting down roots in rural spaces. To this end we consider two ethnographic cases, two life trajectories, of a man and a woman. They are coffee producers in southern Chiapas (Soconusco region) and belong to a generation, born around 1950, who lived two distinct historical moments: an initial socialization structured around agrarian production and the ejido; and beginning in the 1990s, in a new phase of their families' life trajectories, this generation faces de-agriculturalization and individualization of the labor market and state intervention. The paper reveals the weight of gender roles in family solidarities, because men and women do not inherit the same resources nor the same obligations within families.

Key words: rural communities, ejido, family, gender inequalities.

\footnotetext{
* Alicia Rinaldy. Doctora en sociología por la Universidad Sorbonne Nouvelle Paris 3, Francia. Investigadora asociada al Centro de Investigación y Documentación sobre las Américas (CREDA-UMR7227), Francia. Temas de especialización: sociología de las clases populares, sociología de la familia, sociología del género, estudios rurales, metodología y epistemología de la investigación cualitativa. Correo electrónico: alicia. rinaldy@gmail.com. ORCID: https://orcid.org/0000-0002-4932-7608
}

Enviado a dictamen: 17 de febrero de 2020

Aceptación: 8 de diciembre de 2020 


\section{Introducción}

Todos hemos vivido dentro del ejido pero de distintas formas. A unos les fue bien, a otros mal. Otros ya recibieron todo en charola de plata y otros no. Otros empezaron de abajo trabajando. [...] Hay gente que tenía más... Y hay gente que tiene esta ambición de hacer. [...] Y hay unos que dejaron todo a sus hijos y a esos hijos ya no les costó... [...] Y otros no. Otros empezaron a trabajar como jornaleros. Hicieron su capital. Compraron su pedacito. De este hicieron más, poco a poco... Hay muchas diferencias entre todos. No a todos nos fue igual... (entrevista a Consuelo, 2012).

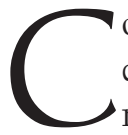

on estos términos Consuelo concluyó su relato de vida que me compartió en el ejido El Paraíso, al suroeste del estado de Chiapas, en el Soconusco (ver Mapa 1). Al ubicar su trayectoria en el panorama más amplio de los habitantes del pueblo, me describió cómo entendía la diferenciación social en su pueblo. Siguiendo su invitación, este articulo contribuye a poner en evidencia los recursos necesarios para quedarse en su pueblo de origen y a cuestionar sus jerarquías internas. Puede ser tentador considerar quedarse en el pueblo de origen como un hecho exclusivamente residual, es decir, como una "inmovilidad forzada". En este sentido, "los que se quedan" serían los que no tienen suficientes recursos (o capitales) socioeconómicos para migrar o los que recibieron las tierras paternas, y por lo tanto la obligación de quedarse. Este artículo contribuye a deconstruir estas hipótesis. Las mujeres entrevistadas habían construido sus vidas en El Paraíso sin recibir tierras ejidales, como "herederas secundarias o residuales” (Deere y León, 2000:336), porque las mujeres reciben la tierra solamente si no existen otros herederos. De la misma manera, algunos hombres que habían sido excluidos de este patrimonio aún permanecieron en el pueblo, mientras otros se fueron a pesar de haber recibido las tierras familiares. En otras palabras, la perpetuación del arraigo en el pueblo no se limita a la herencia de las tierras. Para las clases bajas, la investigación en términos de "capital" tendió a resaltar su ausencia o su escasez en el ámbito económico, social o cultural. Con respeto al análisis del arraigo local y de las solidaridades familiares, esta investigación, a través de los relatos de vida, contribuye a pensar las clases marginadas no exclusivamente bajo el prisma de lo que no tienen, en la negatividad de sus existencias, sino también desde sus experiencias y sus estrategias para "arreglárselas".

El propósito de este artículo es doble. En primer lugar, revelar las experiencias de personas de una generación que ahora no vive exclusivamente del trabajo agrícola, mientras que sus padres se habían beneficiado de la Reforma Agraria y del capitalismo industrial que favorecía la pequeña agricultura campesina. ${ }^{2}$ En segundo lugar, el análisis muestra cómo las solidaridades familiares están profundamente determinadas por el sistema de género y permiten enfrentar los nuevos mercados laborales y las nuevas formas de intervención pública en las zonas rurales de manera desigual.

Esta investigación dará luz a las trayectorias de hombres y mujeres nacidos alrededor de los años 1950 cuyos padres fueron ejidatarios, fundadores del ejido El Paraíso. ${ }^{3}$ De cierta manera, pertenecen a una generación de "transición" porque han experimentado dos momentos históricos distintos. Entre 1930 y 1970, los ejidatarios se beneficiaron de una economía y una política favorables porque los productos de exportación como el café estuvieron sujetos a importantes ayudas estatales. ${ }^{4}$ Sin embargo, tras la orientación neoliberal de inserción competitiva en los mercados internacionales, que culminó con la firma del Tratado de Libre Comercio de América del Norte (TLCAN) el 1 de enero de 1994, o con la política de austeridad, que inauguró el programa de ajuste estructural suscrito con el Fondo Monetario Internacional (FMI) a partir de 1983, el dispositivo público de ayuda a la agricultura se debilitó considerablemente. Los apoyos económicos para el sector agrícola se otorgan ahora en su mayoría a través de programas ${ }^{5}$ para productores competitivos, integrados en los mercados agrícolas internacionales, mientras poco a poco los pequeños productores que no pudieron cumplir con las metas de estos programas se volvieron beneficiarios de políticas de reducción de la pobreza. El Estado considera al pequeño productor de 
las regiones del sur ya no como campesino, sino como "pobre" (Merklen, 2003). Ya no será beneficiario de políticas agrícolas para la inversión - como Alianza para el Campo, la Agencia de Servicios a la Comercialización y Desarrollo de Mercados Agropecuarios (ASERCA) o el Programa de Apoyos Directos al Campo (PROCAMPO) - , sino de programas de lucha contra la pobreza -el más emblemático de estos dispositivos es el Programa de Educación, Salud y Alimentación (Progresa), renombrado primero Oportunidades y luego Prospera-: 6

[...] los municipios más pobres de México reciben solo una parte modesta de los fondos públicos para la agricultura, solo 6.9 \% del gasto en 2007 se asignó a aquellos con niveles de marginación "muy altos", con otro $30.6 \%$ que iba a aquellos que se consideraban de marginación "alta", aunque en conjunto representaban $57.5 \%$ de las unidades de producción que recibían apoyos (Fox y Haight, 2010:46).

En este contexto, se ha observado también un proceso de "desagrarización": no es que la agricultura haya desaparecido completamente de los espacios rurales, sino que su participación en la economía de estos territorios ha disminuido de manera muy significativa (Escalante et al., 2008; Grammont, 2009; Salas y González, 2013; Torres, 2012), además de que las familias combinan trayectorias laborales más diversas, más terciarias y menos agrarias.

En la primera parte de este texto presento los conceptos claves del análisis - solidaridad familiar con perspectiva de género- - y la metodología cualitativa usada para la investigación —encuesta etnográfica y relatos de vida-, a la vez que revisaré los cambios de perspectiva que se han dado en el campo académico para analizar las solidaridades familiares en las zonas rurales mexicanas. En las partes segunda y tercera me introduciré en la historia íntima de dos familias para tratar de entender cómo circulaban las protecciones relacionadas con el ejido cuando los encuestados aún pertenecían a la primera generación de familias ejidales —etapa de fusión-, antes de formar su propia familia —etapa de fisión y nueva fusión-. Mostraré cómo estos recursos permiten enfrentar nuevas situaciones laborales y nuevas formas de intervención pública en el pueblo. Las familias encuestadas presentaban trayectorias laborales menos agrarias, más diversas - a través de la pluriactividad-, más terciarias y fuertemente diferenciadas según los individuos, así como el soporte familiar del que procedían y del que disponían. En las familias, hombres y mujeres no heredaban los mismos recursos y obligaciones; por lo tanto, se beneficiaban de solidaridades profundamente desiguales.

\section{El marco teórico y metodológico de la investigación}

\section{Genealogía de la investigación sobre las familias rurales en México}

En un primer momento, desde el campo académico las familias rurales eran entendidas más que nada como familias campesinas, y el objetivo era analizar la unidad de producción y consumo que formaban. Desde este punto de vista, los análisis mostraron que, si la familia aseguraba su reproducción a través de la producción agrícola, su organización se basaba en la solidaridad de cada uno de sus miembros para llevar a cabo el trabajo agrícola (enfoque funcionalista). El grupo doméstico y la explotación agrícola eran las dos caras de la misma moneda. Los estudios del economista ruso Alexander Chayanov (1974[1925]) ejercieron una fuerte influencia sobre la manera de concebir las familias campesinas, evidenciando las "leyes que [regulaban] la estructura interna de la economía campesina" (Bartra, 1974:50). La producción agrícola se beneficiaba de una mano de obra familiar y gratuita. Chayanov mostró que el tamaño de la familia, la proporción de sus miembros en capacidad de trabajar en la parcela — distinguiendo de esta manera al consumidor-trabajador del simple consumidor (los niños menores) - y la evolución de la composición de la familia de acuerdo con sus ciclos domésticos (formación, disolución), determinaban la producción agrícola familiar. En otras palabras, la estructura interna de la familia definía las condiciones de la producción agrícola 
y, por lo tanto, su reproducción social. La familia rural se percibía como "una organización de gran cohesión social" (Sevilla y Pérez, 1976:23) y se entendía como una unidad económica basada principalmente en las relaciones de parentesco. Los estudios pioneros de Lourdes Arizpe (1978) contribuyeron a visibilizar considerablemente el papel de las mujeres en las zonas rurales, y contribuyeron a perpetuar la imagen anterior de una familia unida y armoniosa en las zonas rurales. Arizpe mostró cómo la familia encontró en sí misma, a través de su organización y del sistema de apoyo mutuo, las condiciones para su propia reproducción. En ese momento, la división sexual del trabajo en la organización doméstica se consideraba en términos de complementariedad de los roles de género.

Estas perspectivas no permitían ver la jerarquía intrafamiliar, las desigualdades de género y generacionales, ni las conexiones entre el espacio doméstico y los mercados de trabajo rurales. En la renovación de la mirada hacia las familias rurales, cabe destacar al menos tres cambios epistemológicos importantes derivados de las perspectivas de género (Arias, 2009; Ariza y Oliveira, 2001): la dinámica intrafamiliar se concibe en términos de tensiones y relaciones de poder; las actividades domésticas de las mujeres se articulan con sus actividades extradomésticas, y la familia ya no se entiende solo como una unidad de producción y de consumo, sino también como un espacio de socialización y de producción de identidades de género.

En primer lugar, la perspectiva de género permite revelar el carácter fundamentalmente conflictivo del contexto familiar y de las relaciones de dominación entre hombres y mujeres, o entre generaciones. En segundo lugar, esta perspectiva pretende romper con la dicotomía público/privado. Paradójicamente, el ingreso de las mujeres al mercado laboral ha permitido visibilizar el trabajo que realizaban en el espacio doméstico. Tuvieron que salir de los hogares para que se entendiera la importancia de su trabajo no remunerado en la esfera privada, plenamente funcional a la reproducción familiar y a la producción agrícola (Arias, 2003, 2009; De Barbieri, 1984; García y Oliveira, 1994; Rodríguez y Cooper, 2005; Sánchez, 1989; Wainerman, 2000). Esta visión más holística del trabajo de las mujeres permitió entender los vínculos entre el mercado laboral y el espacio familiar. Finalmente, en el análisis sobre el ámbito doméstico, los estudios de género se centraron en la dimensión sociocultural de la familia, entendida como una institución "productora de sentidos y valores estratégicos para la sociedad, tales como los significados del matrimonio, la maternidad, la paternidad ola lealtad filial, pero también a su papel sancionador de los roles sociales existentes" (Ariza y Oliveira, 2004:10). En otras palabras, se trata de examinar cómo se interiorizan en las familias las maneras de pensar y actuar ubicadas del lado "masculino" o "femenino". En el contexto rural, varios estudiosos y estudiosas cuestionaron el orden de género que se construye y redefine en las familias (Arias, 2003; Ariza y Oliveira, 2001; Arriagada, 2007; González, 2003). La reflexión de este artículo se inscribe en la continuación de estos tres enfoques.

\section{¿Solidaridades familiares o desigualdades de género?}

El análisis de las solidaridades familiares se limita a menudo a entender de qué forma son efectivas, de qué modo el apoyo de los familiares permite una mayor protección. Sin embargo, relatar exclusivamente las historias de solidaridad contribuye a presentar la familia como una institución homogénea, que desarrolla estrategias de supervivencia en una lógica casi mecánica. Parece importante matizar esta visión y considerar otras trayectorias en las que los vínculos no son tan fuertes o la influencia de la solidaridad familiar es más restringida. Como bien lo describía Consuelo, la solidaridad familiar es profundamente desigual entre las familias y, dentro de ellas, entre los individuos que las componen. Por lo tanto, la familia no puede ser percibida exclusivamente como un espacio de ayuda mutua donde todos los recursos se comparten de manera equitativa; es también un espacio atravesado por relaciones de tensión, dominación y conflicto, profundamente determinado por el sistema de género. En el marco de esta investigación, se considera la familia tanto desde los recursos que puede aportar, su dimensión "protectora", como desde la complejidad 
de sus relaciones internas, su dimensión "opresiva" (Weber, 2013:34). Se tratará de entender las desigualdades entre las familias y dentro de ellas a través de las relaciones, las obligaciones y los roles de género.

Al explorar las "solidaridades familiares", los investigadores e investigadoras observan a menudo las desigualdades intrafamiliares e interfamiliares, entonces, ipor qué es relevante mantener este término para el análisis? En un número reciente de la revista francesa Questions Féministes, en el artículo introductorio se identificaban por lo menos tres razones para no abandonarlo (Palazzo-Crettol et al., 2018). En primer lugar, porque permite visibilizar el trabajo solidario del cual se hacen cargo las mujeres, además de que también es una herramienta para tomar en cuenta la "agencia" de las mujeres y no considerarlas exclusivamente como víctimas del sistema de dominación intrafamiliar. Así, se entiende la familia no solo en términos de coerción y obligación, sino también como un espacio de empoderamiento para las mujeres. Finalmente, la noción permite pensar la familia como un colectivo de solidaridad, y en este sentido como un lugar probable de emancipación. El desafío político sería por lo tanto hacer más igualitarias las solidaridades familiares y luego poder extenderlas más allá de la esfera privada. Bajo esta perspectiva, la antropóloga argentina Rita Laura Segato sugiere un cambio en la consigna feminista de los años setenta: "lo privado es político", y propone, al contrario, "domesticar la política” (Segato, 2016:25), es decir, desburocratizarla, humanizarla e impulsar lazos de solidaridad y reciprocidad característicos de la sociabilidad de las mujeres, entendida esta última como una construcción social formada por la experiencia de las mujeres, precisamente por la división sexual del trabajo.

Entendiendo las solidaridades en cada familia a través de su "economía oculta", a continuación analizaré los recursos económicos y relacionales, comprendidos como un intercambio de capital social, de información o de relaciones estratégicas en el pueblo (Déchaux, 2007), y examinaré también los “circuitos” en los cuales circulan estos recursos dentro de la misma familia. Estos circuitos de solidaridades difieren según las familias, ya que pueden pasar por los vínculos inter o intrageneracionales o corresponder más a la familia extendida o a la unidad doméstica.

\section{Metodología de la investigación: análisis de dos casos etnográficos}

La encuesta etnográfica se realizó en el ejido El Paraíso durante un año, entre 2012 y 2014, con el uso de varios métodos cualitativos: observaciones, entrevistas, historias de vida y genealogías familiares. Este artículo se basa principalmente en las historias de vida de hombres y mujeres, productores de café, nacidos alrededor de los años 1950. Para Pineau y Le Grand (2002); este método cualitativo tiene tres virtudes principales: se presenta como una necesidad antropológica (entendida como propia al ser humano), es universal (todo el mundo tiene una historia de vida) y, por último, favorece la narración y permite alejarse de los procesos dialécticos de preguntas y respuestas de las entrevistas tradicionales. En este sentido, este método puede contrarrestar la "violencia simbólica"7 que a menudo existe entre el encuestador y los encuestados, valorando los conocimientos y las experiencias de estos últimos. Desde esta perspectiva, las pedagogías de emancipación, basadas ante todo en romper las jerarquías en el conocimiento, han utilizado ampliamente este método para resaltar los saberes del alumnado: cada persona tiene conocimiento, es sujeto de conocimiento, tiene una historia, una visión y una comprensión del mundo (Brun, 2001: Coulon y Le Grand, 2000).

Este artículo se basa en los relatos de vida de dos informantes, Francisco y Leona. Sus trayectorias no corresponden a tipos ideales en el sentido weberiano, es decir, una tipología que para los fines de la investigación acentúa deliberadamente ciertos rasgos de la realidad. No se pretenden ilustrar de manera exhaustiva las distintas formas de solidaridades familiares, pero sus relatos contribuyen a ejemplificar las diferentes facetas de las experiencias en el mundo rural, tanto de los hombres como de las mujeres. Retomando la terminología de la socióloga francesa Florence Weber, considero los dos relatos de vida sobre los que me baso 
como "casos etnográficos" (2013), como se hablaría de "casos clínicos". Su análisis permite explicar cómo cada caso singular se encuentra en la intersección de múltiples historias colectivas. Cada caso etnográfico no es representativo de todas las trayectorias de los hombres o de las mujeres, pero la comparación entre los casos permite destacar lo que parece afectar indiscriminadamente a las trayectorias individuales, los destinos sociales comunes, subrayando al mismo tiempo las diferencias y las singularidades de cada una de las trayectorias recogidas. En otras palabras, el análisis de las trayectorias de Francisco y Leona permitirá entender los procesos que contribuyen a la construcción de las trayectorias de los hombres y de las mujeres del pueblo El Paraíso. ${ }^{8}$

\section{Las solidaridades familiares en clave masculina}

\section{Francisco y el apoyo de sus hermanos}

Para los hombres encuestados, narrar su historia familiar era contar la historia de su familia de origen y el proceso de recepción de las tierras ejidales. A través del relato de Francisco se verá cómo la solidaridad familiar puede usarse para perpetuar la herencia familiar $y$, de esta manera, perpetuar su identidad masculina en el pueblo.

Francisco nació en 1957. Era el tercero de los hermanos. En su familia, estaba tácitamente acordado que sería su hermano mayor, Eliseo, quien recibiría las cinco hectáreas de tierras ejidales de su padre. Sin embargo, la transmisión no ocurrió pacíficamente, porque la lucha por la tierra tuvo lugar dentro mismo de la familia, contra su propio padre:

Mi papa trató de vender todo lo que tenía acá. iTrató de vender hasta la casa! Sí. La casa ya estaba vendida. Y un tío dijo a mi madre: "No te vayas de la casa. ¿Dónde te vas a ir con tus hijos?" Yo era un niño. Y mi mama no se salió. [...] Así que mi hermano se presentó a la asamblea: iba a disputar el terreno en la asamblea. Y vaya que la asamblea apoyó a mi hermano (entrevista a Francisco, 2012).
En 1970 recuperaron dos hectáreas gracias a la asamblea ejidal y, once años después, lograron comprar las otras tres hectáreas. Francisco obtuvo oficialmente los derechos ejidales en 1981. Tenía entonces 24 años y dos niños pequeños, de uno y tres años. En 1969, el hermano mayor de Francisco se fue por primera vez a la Ciudad de México a trabajar en la construcción para ayudar a su madre, embarazada de su séptimo hijo. Fue y regresó varias veces: "Yo también quería irme", me dijo Francisco, "pero él tenía la tierra de mi padre y yo la tenía que cuidar, tenía como 12, 13 años". Los recursos económicos de la familia no fueron suficientes para enviar dos hijos a la gran ciudad. Luego, en 1975, su hermano se mudó a Ecatepec, en el Estado de México. Después de la salida de su hermano Francisco tuvo que administrar la tierra:

Eliseo [su hermano mayor] se fue en los setentas. Yo también quería irme a la ciudad; yo también quería ser alguien... Pero era él o yo. No podíamos ir los dos; no alcanzaba. Él tenía las tierras de mi padre. Y yo las cultivaba mientras estaba en D.F. Un día regresó y me dijo: 'Ya no quiero vivir aquí, no quiero esas tierras, te las doy" [...] Mi hermano se comportó como un padre cuando el nuestro se fue. Y cuando se fue, yo me encargué de mis hermanos (entrevista a Francisco, 2014).

Los otros hermanos mayores de Francisco también viajaron para buscar nuevas oportunidades de trabajo fuera de la unidad de producción familiar y del pueblo de origen, por lo que pudieron apoyar económicamente la producción de café de Francisco. Marcela, su hermana mayor, emigró a Puebla, Alma a Tapachula, y Orlando se fue brevemente a la Ciudad de México con Eliseo antes de establecerse finalmente en Tapachula. Francisco recibió ayuda de su hermano mayor y del marido de su hermana mayor desde Puebla, lo que le permitió comprar nuevas tierras.

Hay por lo menos un elemento decisivo en la historia de Francisco que le permitió superar la primera herencia de la tierra y recuperar sus derechos ejidales: la protección legal y colectiva del ejido - "la Asamblea apoyó a mi hermano"- . Lejos de estar protegidos por la familia - en este caso por el padre-, fue de ella de 
la que tuvieron que protegerse. La institución ejidal tomó el relevo para perpetuar el patrimonio patrilineal de la tierra. En este sentido, el ejido es "una institución suprafamiliar de coordinación entre los jefes de familia de los ejidatarios, que dota de recursos organizativos y normas (así como de la capacidad de hacerlas cumplir) que permitía, entre otros factores, regular las relaciones con sus descendientes" (Léonard, 2004:114, traducción propia), en este caso concreto con su padre. Para asegurar la herencia patrilineal de la tierra, Francisco contó también para su beneficio con la solidaridad de sus hermanos.

\section{Una solidaridad horizontal para recrear el patrimonio familiar}

Durante las entrevistas, cuando los hombres se referían a su familia, hablaban principalmente de su familia de origen. Vincularon su historia a la generación anterior, a los padres fundadores del ejido, al linaje paterno, porque es este linaje el que permite teóricamente la herencia de la tierra. En el medio rural, esta herencia fue "una condición fundamental para la reproducción del hogar, pues el acceso a la tierra es lo que garantiza la continuidad de la unidad de producción y reproducción entre las generaciones" (Deere y León, 2000: 326). Sin embargo, recibir las tierras ejidales no significaba solamente heredar una protección territorial o económica, sino también recibir un elemento que era parte de la estructuración de la identidad masculina que se transmitía de padres a hijos en el pueblo. En definitiva, se heredaba un modelo patriarcal según el cual, al igual que las mujeres, los hombres enfrentaban normas que prescribían modelos de comportamiento y formas de pensar. La "identidad masculina" se entiende en este marco, entonces, como una categoría de percepción, como una construcción social. Ser "producido" socialmente como "hombreejidatario" estaba íntimamente relacionado con la transmisión de la tierra, la actividad agrícola y el papel desempeñado en la política local. La institución ejidal fue de hecho un vector importante de la producción y reproducción de un modelo normativo de masculinidad. Seguía siendo la base de las primeras socializaciones de los hombres entrevistados y, por lo tanto, todavía constituía un elemento estructurante en las biografías. Recibir tierra implicaba inscribirse en el linaje masculino, que permitía a los hombres existir simbólicamente como tales, económicamente como productores y esposos, al ser los principales proveedores del hogar, y políticamente como ejidatarios —al ejercer su ciudadanía agraria en la asamblea ejidal—: "El honor del hombre campesino residía en poseer y trabajar la tierra para mantener a su familia; de ahí que los derechos de propiedad se convirtieran en elemento indispensable para alcanzar un estatus de respeto" (Torres, 2009:462).

La genealogía de Francisco ilustra las dinámicas de migración y de solidaridad de los diferentes miembros de la familia. Con el apoyo de su familia extendida, él pudo consolidar y diversificar sus fuentes de ingresos. Fueron las migraciones y el éxito económico de los miembros de su familia los que le permitieron a Francisco permanecer en el pueblo y continuar cultivando la tierra. En el momento de la entrevista tenía ocho hectáreas, casi había duplicado la herencia paterna; producía alrededor de veinte toneladas de café al año, una cantidad muy importante en comparación con los otros productores del pueblo, y empleaba permanentemente a dos trabajadores agrícolas guatemaltecos en su parcela. También alquilaba un local en el pueblo y un taxi que hacía cotidianamente viajes entre la ciudad de Tapachula y El Paraíso. La pluriactividad le permitía mejorar los ingresos del hogar e invertir en la producción agrícola. Este mecanismo lo mantenía en un proceso de reproducción social, a la vez que le permitiría satisfacer las necesidades de las generaciones siguientes (Piñeiro y Cardeillac, 2010). En esta familia era el apoyo mutuo entre hermanos y hermanas de la misma generación lo que finalmente les permitió una inserción laboral y asegurar también el legado de antiguas formas de masculinidad. Al recibir tierras y ayudas económicas, Francisco pudo convertirse a su vez en un apoyo para las generaciones posteriores y rehabilitar una identidad masculina en peligro en un primer momento - por el conflicto derivado de las parcelas de su padre- .

La solidaridad familiar que experimentó Francisco se trató, ante todo, de una "solidaridad horizontal" 
basada en los lazos de hermandad. Durante el trabajo de campo, encontré otros tipos de familias con solidaridades familiares mucho más "verticales", como en el caso de la historia de Álvaro. Este, a finales de la década de los ochenta, recibió la totalidad de la dotación de su abuelo, cinco hectáreas. En el momento de la entrevista tenía tres hijos. Uno era guardia de seguridad privada, otro obrero en una conservería de Tapachula y el último, taxista en el pueblo: "Tuvieron que buscar otra forma de vivir aquí, trabajar en algo que no sea la tierra para apoyarnos" (entrevista a Álvaro, 2014). Ayudaban económicamente a sus padres, pero no invertían en la parcela paterna. Álvaro se volvió dependiente de sus hijos, que ya no consideraban las tierras ejidales como parte del patrimonio familiar o como un recurso rentable (Del Rey, 2004; Del Rey y Quesnel, 2007). En este caso, el apoyo de los hijos a Álvaro constituía una solidaridad construida "desde abajo" o "solidaridad ascendente". Sin embargo, él lo experimentaba de manera problemática, porque quedaba en evidencia su incapacidad para mantener a la familia a través de la producción de café. Para muchos, especialmente para quienes no pudieron diversificar sus fuentes de ingresos, la tierra ya no era un recurso valioso y valorado en el pueblo.

\section{Las solidaridades familiares en clave femenina}

\section{Leona: una solidaridad ascendente}

El acceso a los recursos territoriales, económicos, políticos o relacionales depende de los vínculos, de la naturaleza de estos vínculos y del lugar ocupado en la familia como hijo o hija de ejidatarios. Las mujeres entrevistadas a menudo estaban menos recursos que los hombres, ya que eran las "herederas residuales" (Deere y León, 2000:336) de las tierras ejidales de sus padres. Recibían tierra si no había otros herederos, y, en caso de recibir alguna, el número de hectáreas era inferior al de sus hermanos - un promedio de 4.5 hectáreas para los hombres, contra 2.3 hectáreas para las mujeres en el contexto de esta investigación-. Me referiré a la historia de Leona, cuya trayectoria es radicalmente diferente a la de Francisco por ser mujer, porque no heredó la tierra de sus padres y porque tuvo que irse para apoyar a su familia.

Leona nació en 1966. Cuando su padre murió, su abuela la cuidó a ella y a su hermana menor, Elvira. Su hermano heredó las tierras ejidales del padre. A los 15 años, a principios de los ochenta, Leona se fue a la Ciudad de México para trabajar y apoyar a sus abuelos, a quienes enviaba parte de su sueldo. Después de ocho años, regresó finalmente a su pueblo para cuidar a su abuela. Se casó poco tiempo después y enseguida nació su primera hija, Maricel. En ese momento, abrió con su marido una tienda de abarrotes. Al momento de la entrevista Leona estaba separada de su marido y manejaba la economía familiar con la ayuda de sus dos hijas. Hacía aproximadamente cinco años, compraron dos hectáreas de tierra ejidal. Leona era ejidataria:

iSon las chamacas! Ellas vendieron zapatos, venden cursos de costura... Y ellas fueron las que ayudaron a comprar el terrenito. Fue con ellas. Con la tienda Andrea (venta a domicilio). De ahí juntaban y de ahí compramos... Con lo poquito que sale se da para limpiar, los trabajos... Y lo demás... (entrevista a Leona, 2012).

Ganaban treinta pesos por cada par de zapatos que vendían. Su hija mayor, Maricel, era profesora de costura. En 2012, trabajaba en dos programas en pueblos vecinos: en el programa Artesanos de la Secretaría de Desarrollo Social (SEDESOL) y en el Programa para Mejoramiento de la Producción y Productividad Indígena de la Comisión Nacional para el Desarrollo de los Pueblos Indígenas. Sin embargo, una vez concluyeron las clases de costura en noviembre de 2013, Maricel no contó con trabajo fijo. Su segunda hija, Adela, trabajaba en el mercado de Tapachula con una prima, donde vendía especies. Finalmente, contaban como fuente de ingreso en su hogar con subsidios públicos, en concreto recibían apoyo del programa Oportunidades, que les permitía pagar las múltiples idas y vueltas de la familia a Tapachula. 


\section{Estar al servicio de la familia}

Durante las entrevistas, las mujeres hablaron con más detalle de su historia familiar. Como escribió Richard Hoggart en relación con las clases populares inglesas, las mujeres son "las historiadoras de la familia" (1991:42), precisamente porque están al servicio de ella, y tienen la experiencia práctica y cotidiana del parentesco. En este sentido, en la intimidad de las familias, la división sexual no ocurre exclusivamente en relación con las tareas domésticas, sino también con el conocimiento genealógico, que es un reflejo y una extensión de las mismas. En otras palabras, este conocimiento revela el uso social diferenciado que se hace de la familia y no las capacidades de memoria de los individuos (Le Wita, 1988). Entender las maneras de definir la familia es entender lo que es socialmente importante para los individuos que participan en ella. En este sentido, las mujeres hablaban con mayor detalle de sus familias y sus relatos se centraban en la familia que habían formado, no en la familia de origen, a diferencia de los hombres que hacían más referencia a esta última. Sin poder esperar algo de la generación anterior, las mujeres encuestadas estructuraban sus narrativas alrededor de su "carrera matrimonial" y luego de su maternidad, porque el matrimonio era para ellas la esperanza de vivir mejor con su pareja, y el hombre era considerado como el principal proveedor del hogar.

Leona no recibió las tierras de su padre o de su esposo, de quien se divorció. Ella migró a la Ciudad de México a principios de la década de los ochenta, lo que le permitió ayudar a sus abuelos, y regresó cuando ellos enfermaron. A diferencia de Francisco, Leona no recibió tierras de la generación anterior, pero le corresponde a ella apoyar a los miembros de esta generación: a sus abuelos. Sin la herencia de la tierra, sin la presencia de un marido, Leona y sus hijas trabajaron juntas para reconstruir un patrimonio familiar del cual fueron inicialmente excluidas. De entonces en adelante, el nuevo contexto rural les permitió diversificar las fuentes de ingresos para el hogar; ya no necesitaban migrar a la "gran ciudad" para encontrar oportunidades de trabajo, como se solía hacer durante el período histórico anterior. Las fuentes de ingresos de la familia eran múltiples: la tienda de abarrotes, la venta de zapatos a domicilio, las clases de Maricel, el trabajo de Adela en el mercado de Tapachula, los subsidios públicos y las tierras de café. Al igual que Francisco, Leona recurrió a la pluriactividad; sin embargo, y a diferencia de él, compartía esta pluriactividad, que además era incierta y precaria, con sus hijas: la tienda no era tan rentable como lo había sido antes; las clases de costura se interrumpieron; el trabajo agrícola era incierto por definición y resultaba poco rentable dado que solo contaban con dos hectáreas, y no siempre era fácil "encontrar la buena puerta", como dijo, para acceder a los subsidios públicos.

La solidaridad familiar horizontal de Leona era mucho más restringida que la de Francisco. Las relaciones con su familia extendida se limitaban a una hermana que vivía en Tapachula. También su reducido capital social le dificultaba el acceso a los programas del gobierno. De hecho, encontrar la "buena puerta" consistía a menudo en tener buenas conexiones para lograrlo, en tener un capital relacional más amplio. Por el contrario, Marcia, la esposa de otro ejidatario encuestado, trabajaba todas las mañanas en el colegio del pueblo, donde preparaba el desayuno para maestros y alumnos. Tuvo esta oportunidad de trabajo gracias a su esposo, miembro del comité ejidal, quien la puso en contacto con personal de las oficinas de la SEDESOL en Tuxtla Gutiérrez. Después de tres intentos, se incorporó al programa Cocinas Comunitarias. En este sentido, cuanto más cerca están los individuos de los ejidatarios o del comité ejidal, ente que aún congrega el poder en el nivel local, más están informados de los programas a los cuales pueden postular. Más allá de obtener una ayuda directa, por ejemplo, de saber cuándo hay reuniones de información incrementa las posibilidades y la gama de ofertas, así como las opciones para moverse fácilmente entre las distintas secretarías y jugar con las características requeridas de cada programa. En otras palabras, es el acceso al capital social, entendido como intercambio de información o de relaciones estratégicas, lo que condiciona el acceso a las políticas públicas. 
La historia familiar de Leona ilustra cómo el mismo patrón de apoyo familiar se perpetúa de una generación a otra. En general, esta solidaridad, construida desde abajo, es ascendente — cuando las generaciones más jóvenes ayudan a las más viejas-, y se trata del tipo de solidaridad que experimentaron por lo común las mujeres madres solteras del pueblo, como me lo señaló otra de las encuestadas: "Con lo poco que tenemos ayudamos a mi padre, porque estamos acostumbradas a esto. Ese es nuestro papel como mujer. Así es" (entrevista a Marcelina, 2012). Sin embargo, esta solidaridad familiar ascendente no se vivía de manera problemática en el caso de las mujeres porque representaba su horizonte social, porque el papel de ellas consistía en estar al servicio de la familia. Si las mujeres no podían contar con una herencia, el legado de su familia de origen, entonces las familias que iban a formar, sus relaciones conyugales o los vínculos con sus hijos e hijas eran cruciales.

\section{Conclusión}

A través de estos dos relatos de vida puede observarse la construcción de distintos tipos de solidaridad familiar según el género. Si algunas familias operan como una protección adicional, una "protección cercana" (Castel, 2003:11), activando la cadena de solidaridad de sus miembros y solicitando la ayuda mutua de los hermanos, de las generaciones anteriores o siguientes, otras no tienen recursos suficientes para preservar a las personas de las múltiples inseguridades de la vida social. El acceso a los recursos territoriales, económicos, políticos o relacionales del ejido depende de los vínculos, de la naturaleza de estos enlaces y de la posición ocupada en la familia como hijo o hija. Por lo tanto, la solidaridad familiar adopta formas y contornos muy diferentes en función de los recursos - económicos, relacionales, de tierras y de las posiciones de poder en el pueblo- presentes en cada familia y de las características sociodemográficas de cada individuo, como el género o el orden de nacimiento en la familia. Además, si bien las historias vinculadas con sus matrimonios fueron decisivas para las mujeres, las características de la familia de origen fueron más determinantes para los hombres.

Francisco pudo compensar la herencia de la tierra que se dificultó por causa de su padre gracias a la protección de la institución ejidal, pero también a través de una "solidaridad horizontal", entre colaterales, que le permitía formar parte de un linaje masculino y, por lo tanto, existir simbólicamente como hombre en el pueblo. Porque "producirse" socialmente como hombre en el pueblo estaba íntimamente ligado a la transmisión de la tierra y a la actividad agrícola. El ejido fue, en efecto, una institución importante para la producción y reproducción de un modelo normativo de masculinidad; fue el soporte de las primeras socializaciones de los hombres encuestados y, por lo tanto, todavía, un elemento estructurador de las biografías. Sin embargo, este soporte, sobre el cual se construyeron las identidades masculinas de padre, esposo, productor y ejidatario, se debilitó. Si los hombres hablaban de la dificultad de vivir de su producción de café, esta dificultad era individualizada y se culpaban de no poder mantener la economía familiar por sí solos. Por otro lado, la historia familiar de Leona mostró cómo las solidaridades familiares pueden dibujar otros patrones y responder a otras lógicas de ayuda mutua entre mujeres: una solidaridad ascendente, por la que las nuevas generaciones ayudan a las anteriores, reforzando el trabajo femenino al servicio de la familia. Por ser mujeres no podían contar con una herencia familiar —o solo de manera residual- y tenían que apostar por el éxito de sus relaciones como madres, y eventualmente como esposas.

Desde hacía unos veinte años, el programa Progresa — antes llamado Oportunidades y Prospera - había convertido a las madres de zonas marginales en receptoras de subsidios monetarios. Contribuyó también a estructurar las zonas rurales y a hacer de las mujeres las nuevas intermediarias políticas en las comunidades. En 2011, más de 5.8 millones de familias recibieron el programa en todo el país: el $62.4 \%$ se ubicaban en zonas rurales, el $20 \%$ en zonas urbanas y el $17 \%$ en zonas semiurbanas. Las regiones que más se beneficiaron de este programa fueron las del sur del país, en particular el 
estado de Chiapas, donde se encontraban más del $10 \%$ de los beneficiarios nacionales (SEDESOL, 2012). En 2019, bajo la administración del presidente Andrés Manuel López Obrador, este programa fue desmantelado de modo que, de los tres componentes de la intervención —salud, alimentación y educación-, solo quedaron las becas escolares, el programa Becas para el Bienestar Benito Juárez. Entre las críticas que podrían formularse hacia esta política pública de inspiración neoliberal, su reestructuración representa una importante pérdida de ingresos y de poder para las mujeres. Es muy probable que esta pérdida en los presupuestos familiares ejerza una mayor presión sobre las ya precarias solidaridades familiares en clave femenina.

\section{Notas}

${ }^{1}$ El nombre del pueblo y de los entrevistados fueron cambiados para preservar el anonimato.

${ }^{2}$ El modelo económico de sustitución de importaciones, característico del capitalismo industrial en América Latina, generó una fuerte industrialización. En este contexto, la agricultura proporciona grandes cantidades de alimentos baratos para alimentar las ciudades y se vuelve funcional al desarrollo de este proceso de industrialización (para profundizar en esta perspectiva, ver: Rubio, 2003).

${ }^{3}$ La dotación de tierras se llevó a cabo en 1943.

${ }^{4}$ El Instituto Mexicano del Café (INMECAFE), creado en 1958, apoyaba a los productores de café, les otorgaba créditos con una tasa de interés muy baja, les proporcionaba asistencia técnica a través de la distribución de árboles y gestionaba la comercialización del café con la compra del producto a precios de garantía. También proporcionaba a los productores una serie de servicios, como el seguro social o la construcción de infraestructuras en los pueblos: escuelas, carreteras, electricidad, centros de salud, etcétera.

${ }^{5}$ Como PROCAMPO, una ayuda directa a los productores según el número de hectáreas cultivadas, o ASERCA para la comercialización de la producción.

${ }^{6}$ Proporciona subsidios monetarios a las madres de familia en áreas marginales, principalmente rurales.
7 "La violencia simbólica es esa violencia que arranca sumisiones que ni siquiera se perciben como tales apoyándose en unas 'expectativas colectivas', en unas creencias socialmente inculcadas" (Bourdieu, 1999:173).

${ }^{8}$ Para leer otros casos etnográficos, los lectores están invitados a consultar la tesis doctoral en la que está basado este artículo (Rinaldy, 2016).

\section{Referencias}

Arias, Patricia (2003). "Diversidad rural y relaciones de género en México, ayer y hoy”. En Estudios del hombre; 17(1), p. 1546.

Arias, Patricia (2009). Del arraigo a la diáspora, dilemas de la familia rural. México: Universidad de Guadalajara, Porrúa.

Ariza, Marina y Orlandina de Oliveira (2001). "Familias en transición y marcos conceptuales en redefinición”. En Papeles de Población, 28(1), p. 939.

Ariza, Marina y Orlandina de Oliveira (2004). Imágenes de la familia en el cambio de siglo. México: IIS-UNAM.

Arizpe, Lourdes (1978). Migración, etnicismo y cambio económico (un estudio sobre migrantes campesinos a la ciudad de México). México: El Colegio de México.

Arriagada, Irma (2007). "Familias latinoamericanas: cambiantes, diversas y desiguales". En Papeles de Población; 53(1), p. 922.

Bartra, Roger (1974). Estructura agraria y clases sociales en México. México: Era.

Bourdieu, Pierre (1999). Razones prácticas. Sobre la teoría de la acción. Barcelona: Anagrama.

Brun, Patrick (2001). Emancipation et connaissance. Les histoires de vie en collectivité. París: L'Harmattan.

Castel, Robert (2003). L'insécurité sociale. Qu'est-ce qu’être protégé? París: Seuil.

Chayanov, Alexander (1974[1925]). La organización de la unidad económica campesina. Buenos Aires: Nueva Visión.

Coulon, Marie Jo y Jean-Louis Le Grand (2000). Histoires de vie collective et éducation populaire: les entretiens de Passay. París: L'Harmattan. 
De Barbieri, Teresita (1984). Mujeres y vida cotidiana. México: FCE, IIS-UNAM.

Déchaux, Jean-Hugues (2007). Sociologie de la famille. París: La Découverte.

Deere, Carmen Diana y Magdalena León (2000). Género, propiedad y empoderamiento: tierra, Estado y Mercado en América Latina. México: PEUG, FLACSO-Ecuador.

Del Rey, Alberto (2004). Movilidad y longevidad en las dinámicas familiares multigeneracionales. Aplicación al medio rural del Sotavento Veracruzano, México. Tesis de doctorado en demografía, Barcelona: Universidad Autónoma de Barcelona.

Del Rey, Alberto y André Quesnel (2007). "Las implicaciones intrafamiliares, intergeneracionales y migratorias de la política agraria en México. El caso del sur del estado de Veracruz, México". En Ulúa. Revista de Historia, Sociedad y Cultura, 9(1), pp. 59-86.

Escalante Semerena, Roberto I., Horacio Catalán, Luis Miguel Galindo y Orlando Reyes (2008). "Desagrarización en México: tendencias actuales y retos hacia el futuro". En Cuadernos de Desarrollo Rural, 59(4), pp. 87-116.

Fox, Jonathan y Libby Haight (2010). Subsidios para la desigualdad. Las políticas públicas del maíz en México a partir del libre comercio. México: Woodrow Wilson International Center for Scholars.

García, Brígida y Orlandina de Oliveira (1994). Trabajo femenino y vida familiar en México. México: El Colegio de México.

González Montes, Soledad (2003). "Las relaciones intergeneracionales y de género en la transición de una economía campesina a una economía diversificada". En Heather Fowler-Salamini y Mary Kay Vaughan (eds.), Mujeres del campo mexicano, 1850-1990. Zamora, México: El Colegio de Michoacán, pp. 273-293.

Grammont, Hubert Carton de (2009). "La desagrarización del campo mexicano”. En Convergencia, 50(1), pp. 13-55.

Hoggart, Richard (1991). 33 Newport Street. Autobiographie d'un intellectuel issu des classes populaires anglaises. París: Éditions du Seuil.

Léonard, Éric (2004). "La réforme agraire mexicaine comme processus de frontière. Logiques d'autono- misation, ancrage de l'État et production institutionnelle dans la région des Tuxtlas". En Autrepart, 30(1), pp. 97-116.

Le Wita, Béatrix (1988). Ni vue, nie connue. Approche ethnographique de la culture bourgeoise. París: Éditions de la $\mathrm{MSH}$

Merklen, Denis (2003). "Du travailleur au pauvre. La question sociale en Amérique latine". En Études Rurales, 165-166(1), pp. 171-196.

Palazzo-Crettol, Clothilde, Carola Togni, Marianne Modak y Françoise Messant (2018). "Les enjeux sexués des ‘solidarités familiales”. En Nouvelles Questions Féministes, 37(1), pp. 8-13.

Pineau, Gaston y Jean-Louis Le Grand (2002). Les histoires de vie. París: PUF.

Piñeiro, Diego E. y Joaquín Cardeillac (2010). "Influencia de la composición del grupo familiar en la pluriactividad”. En Tabaré Fernández (coord.), El Uruguay desde la sociología VIII, Montevideo: Facultad de Ciencias Sociales-Universidad de la República.

Rinaldy, Alicia (2016). Rester au village. Une génération à l'épreuve des changements économiques, politiques et familiaux au sud du Mexique (1943-2014). Tesis de doctorado en Sociología, Institut des Hautes Études de l'Amérique latine, Paris 3 Sorbonne Nouvelle, París.

Rodríguez Chaurnet, Dinah y Jennifer Cooper (2005). Debate sobre el trabajo doméstico: antología. México: Instituto de Investigaciones Económicas-Universidad Nacional Autónoma de México, Escuela Nacional de Trabajo Social, Centro de Investigaciones Interdisciplinarias en Ciencias y Humanidades.

Rubio, Blanca (2003). Explotados y excluidos. Los campesinos latinoamericanos en la fase agro exportadora neoliberal. México: Plaza y Valdés.

Salas Quintanal, Hernán e Íñigo González de la Fuente (2013). "Nueva ruralidad. Procesos sociolaborales y desagrarización de una sociedad local en México (1980-2010)". En Gazeta de Antropología, 29(2).

Sánchez Gómez, Martha Judith (1989). "Consideraciones teóricometodológicas en el estudio del trabajo doméstico en México". En Orlandina de 
Oliveira (coord.), Trabajo, poder y sexualidad. México:

El Colegio de México-Programa Interdisciplinario de Estudios de la Mujer, pp. 59-79.

SEDESOL (2012). Cuarto informe trimestral 2011. México: SEDESOL.

Segato, Rita Laura (2016). La guerra contra las mujeres. Madrid: Traficantes de Sueños.

Sevilla Guzmán, Eduardo y Manuel Pérez Yruela (1976).

"Para una definición sociológica del campesinado". En Agricultura y Sociedad, 1(1), pp. 15-39.

Torres Mazuera, Gabriela (2009). "La territorialidad rural mexicana en un contexto de descentralización y competencia electoral". En Revista Mexicana de Sociología, 7l(3), pp. 453-490.

Torres Mazuera, Gabriela (2012). "El ejido posrevolucionario: de forma de tenencia sui generis a forma de tenencia ad hoc". En Península, 7(2), pp. 69-94.

Wainerman, Catalina (2000). "División del trabajo en familias de dos proveedores. Relato desde ambos géneros y dos generaciones". En Estudios Demográficosy Urbanos, 15(1), pp. 149-184.

Weber, Florence (2013). Penser la parenté aujourd'hui. La force du quotidien. París: Éditions Rue d'Ulm, Presses de l'École normale supérieure.

Mapa 1. El ejido El Paraíso en la zona cafetalera del sur de Chiapas

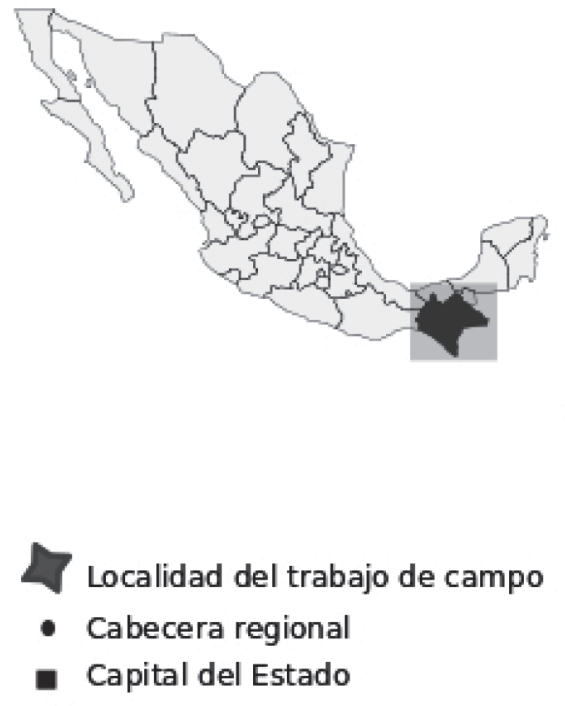

- Soconusco

Fuente: CEIEG, 2013.

A. Rinaldy, 2020, hecho con Philcarto e Inkscape.

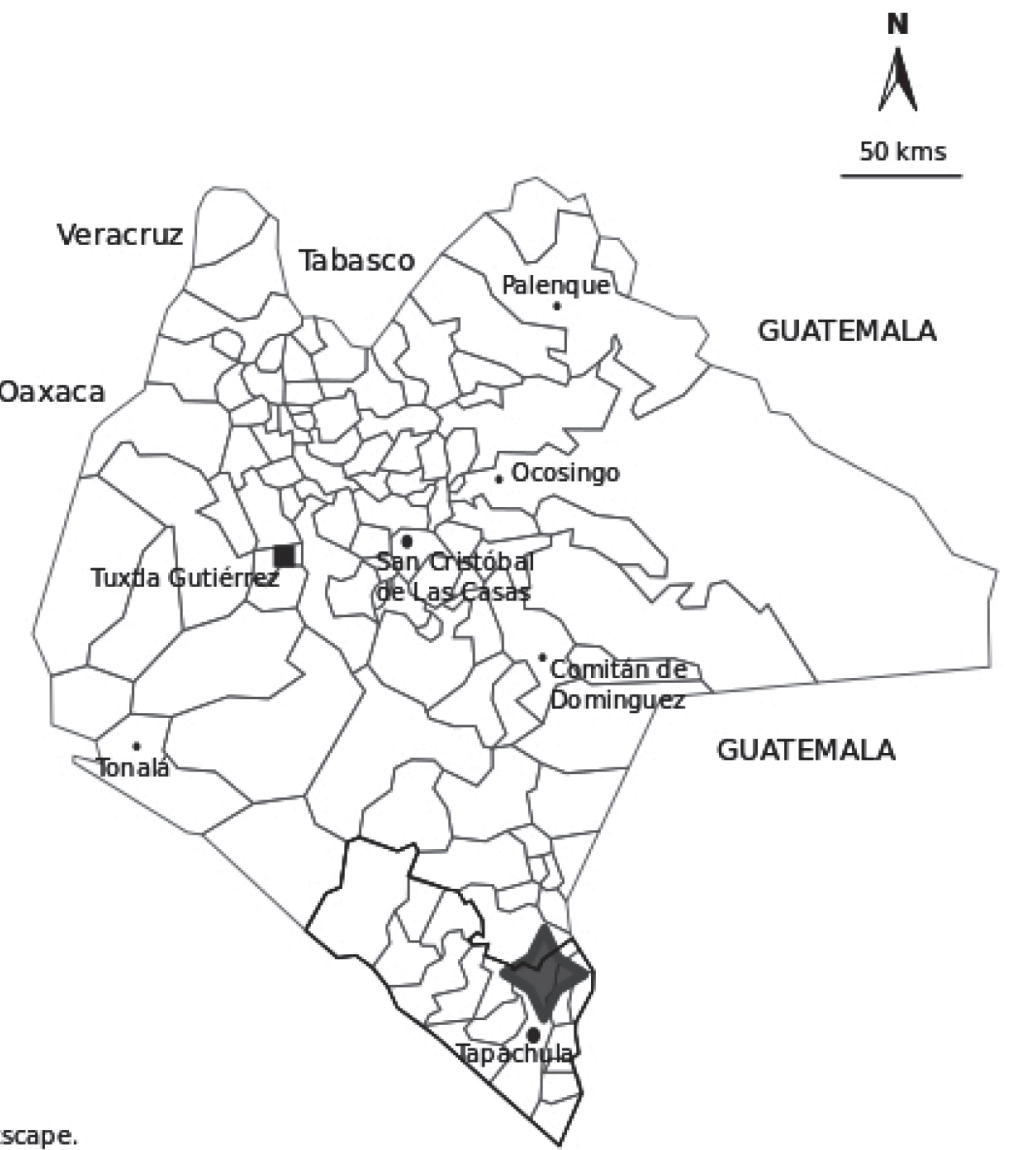

